\title{
A Way to Use Waste Heat to Generate Thermoelectric Power
}

\author{
Marian Brázdil ${ }^{1}$, Jiří Pospíšil $^{1}$ \\ ${ }^{1}$ Brno University of Technology, Faculty of Mechanical Engineering, Energy Institute, Department of Power \\ Engineering, Technická 2896/2, 61669 Brno, Czech Republic
}

Correspondence to: brazdil@fme.vutbr.cz

\begin{abstract}
In recent years there has been rising interest in thermoelectric generation as a potential source of electric power using waste heat. This paper describes thermoelectric power generation from waste heat from biomass boilers, utilizing generators that can convert heat energy directly to electrical energy. General principles of thermoelectric conversion and future prospects of these applications are discussed.
\end{abstract}

Keywords: thermoelectricity, thermoelectric generator, seebeck effect, waste heat.

\section{Introduction}

The efficiency of traditional power generating systems is not satisfactory. A huge amount of thermal energy is discharged into the atmosphere every day. Not all of the waste heat is dispersed in the atmosphere without profit, but a major part of the energy buried in fossil fuels is not converted into electricity. For example, the total energy efficiency of a conventional thermal power plant using steam turbines is approximately $40 \%$; the best modern combinedcycle plant using a gas turbine and a steam turbine is between 50-60\%. In vehicles using gasoline-powered combustion engines the conversion efficiency is about $30 \%$, and diesel-powered combustion engines achieve about $40 \%$ efficiency.

Thermoelectric generators (TEG) have the potential to recover waste heat as effective energy and to make a major contribution to reducing fossil fuel consumption. As a consequence of lower energy consumption and higher total energy efficiency, TEG also can help reduce $\mathrm{CO}_{2}$ and other greenhouse gas emissions.

\section{General principles of thermolectric conversion}

Thermoelectric devices are semiconductor devices based on thermoelectric effects that can convert thermal energy directly into electricity. These solid state devices use electrons as their working fluid.

Thermoelectric effects can be used both for power generation and for electronic refrigeration. These effects are often explained by two different electrically conductive materials connected together. When a temperature gradient is established between junc- tions of materials - e.g. one junction is heated and the other cooled, as shown in Figure 1, a voltage (Seebeck voltage) is generated. The thermocouple that is created can be connected to a load to provide electric power. This phenomenon was discovered in 1821 by J. T. Seebeck, and is called the "Seebeck effect".

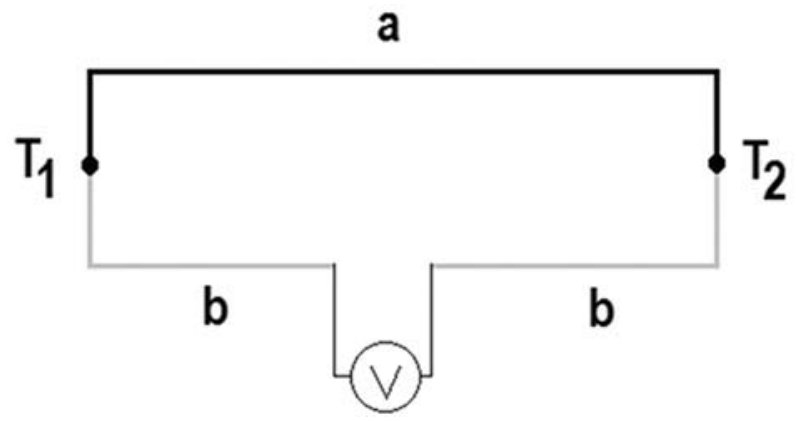

Figure 1: Seebeck effect

The generated voltage $(\mu \mathrm{V} / \mathrm{K})$ is directly proportional to the temperature gradient. The Seebeck coefficient $\alpha$ is a coefficient of proportionality and a material-specific parameter [1].

$$
V_{a b}=\alpha_{a b}\left(T_{1}-T_{2}\right)[\mathrm{V}]
$$

Conversely, it is possible to convert electric energy into a temperature gradient. This complementary phenomenon, known as the Peltier effect, was discovered by C. A. Peltier in 1834. If a voltage is applied across a junction and a direct current flows in the circuit, a slight cooling or heating effect (depending on the direction of the current) occurs at the junction. This effect is extensively used for cooling.

A typical thermoelectric device is composed of a large number of semiconductor thermocouples, see 
Figure 2. Thermocouples consist of n-type and ptype semiconductor pellets connected together with a metal plate by soldering. They generate Seebeck voltage of hundreds of $\mu \mathrm{V} / \mathrm{K}$.

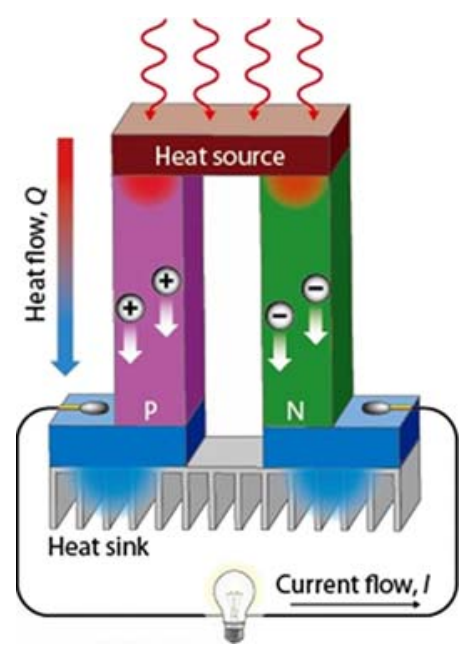

Figure 2: Principle of thermoelectric power generation $[2]$

\section{Thermoelectric materials}

The highest thermoelectric conversion performance is achieved with heavily doped semiconductors. High electrical conductivity $(\sigma)$, large Seebeck coefficient $(\alpha)$ and low thermal conductivity of the materials $(\lambda)$ are necessary in order to realize high-performance thermoelectric materials [3]. The potential to convert heat to electricity is quantified by the so-called thermoelectric figure-of-merit $Z$, which is defined as:

$$
Z=\frac{\alpha^{2} \sigma}{\lambda}\left[\mathrm{K}^{-1}\right]
$$

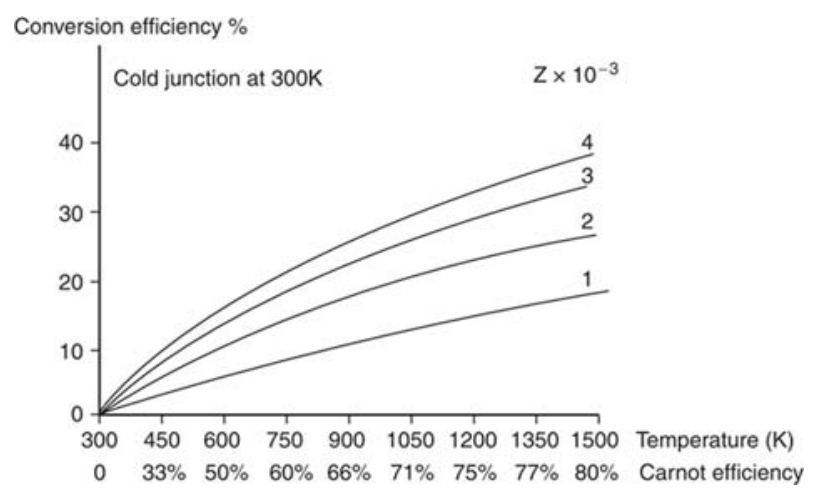

Figure-of-merit $Z$ varies with temperature. As shown in Figure 3, the conversion efficiency is a function of operating temperature difference. An increase in the temperature difference provides an increase in heat available for conversion, so large temperature differences are desirable [3]. It is more usual to specify a dimensionless figure-of-merit, which is equal to $Z T$, where $T$ is absolute temperature.

Only materials which possess $Z T>0.5$ are regarded as thermoelectric materials [3]. Established thermoelectric materials can be divided into groups depending upon the temperature range of operation:

- low-temperature materials, up to around $450 \mathrm{~K}$

- middle-temperature materials, from $450 \mathrm{~K}$ up to around $850 \mathrm{~K}$

- high-temperature materials, from $850 \mathrm{~K}$ up to around $1300 \mathrm{~K}$

Alloys based on bismuth in combinations with antimony, tellurium and selenium are low-temperature materials. Middle-temperature materials are based on lead telluride and its alloys. High-temperature materials are fabricated from silicon germanium alloys [3].

\section{Thermoelectric generators}

A simple thermoelectric generator consists of a series of thermoelectric couples placed between two heat exchangers and a DC/DC converter. Heat from the heat source flows through the thermoelectric couples and is dissipated through the heat sink into the ambient air, as shown in Figure 4. Electric power is generated until a temperature differential is applied. A DC converter changes the output thermoelectric voltage to the voltage required by the load.

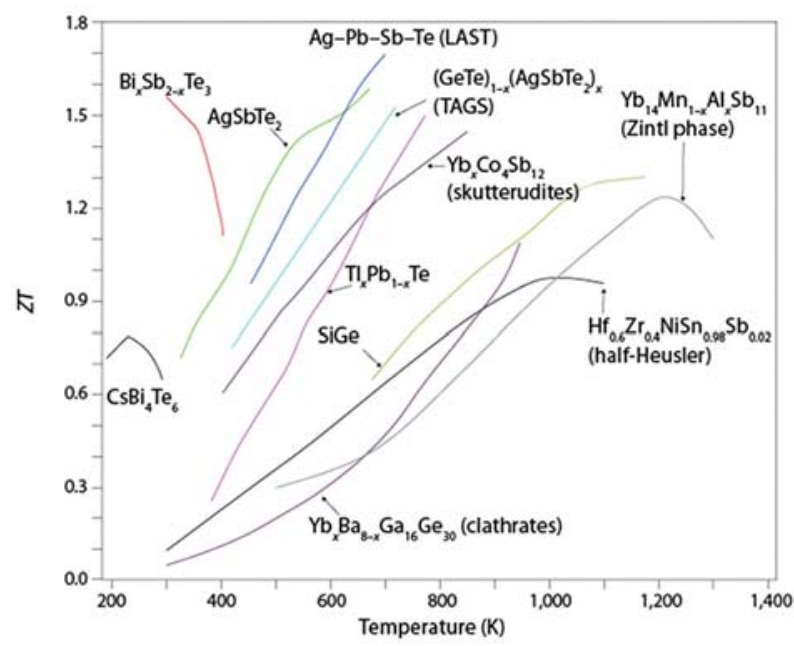

Figure 3: Dependence of conversion efficiency on a given thermoelectric material and the applied temperature difference $[1,2]$ 


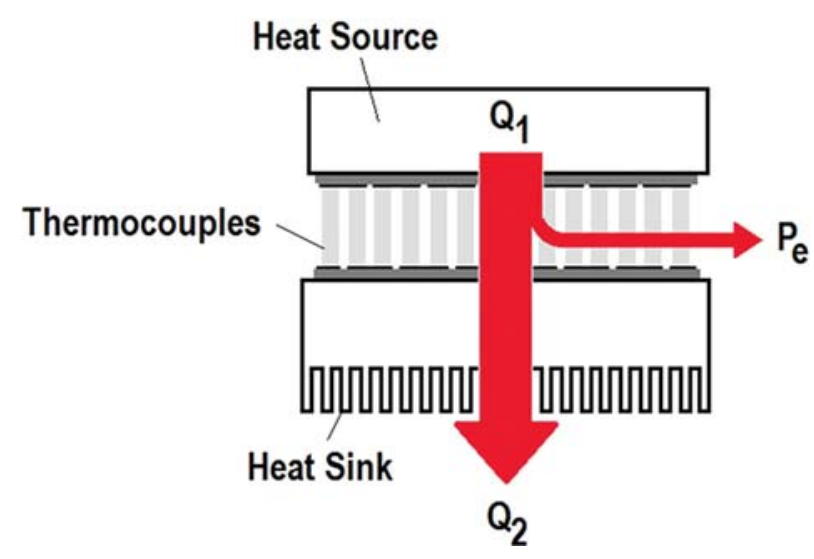

Figure 4: A simple thermoelectric generator

In general, the thermoelectric system offers several advantages: it has no moving parts or chemical substances, has maintenance-free and silent operation, is reliable, and is not position dependent $[1,4]$. On the other hand, the most significant disadvantage is the low energy efficiency of TEG. In addition, the energy efficiency and the released output power are temperature-dependent.

When exploring the universe, radioisotope thermoelectric generators (RTGs) were often used as a power source for deep space probes. Lower energy efficiency was not critical in these applications, because TEGs are suitable for operating in dangerous or inaccessible areas. In ordinary applications, TEGs supplied by combusted fossil fuels (oil or gas) are not cost competitive. Fossil fuels are expensive, so it is better to use waste heat as the energy source.

\section{$5 \quad$ Waste heat from biomass boilers}

The quality and the quantity of various types of waste heat differ. Thermoelectric generators are able to recover waste heat if the heat flux is sufficient. A high operating temperature results in high conversion efficiency.

Modern small-scale biomass combustion systems (pellet boilers) achieve high efficiency and low emissions, and they offer a convenient form of residential heat supply. However, these fully automatic systems require auxiliary electric power for continual fuel supply, $\mathrm{CO}_{2}$ balanced heat production and distribution. These requirements result in dependence on the electricity grid.

TEGs have the potential to deliver auxiliary power for self-sufficient operation of the combustion and heating system [5]. Waste heat from the furnace can be utilised by an integrated generator. In fact, the boiler becomes a micro-scale CHP unit. The thermoelectric generator of the boiler needs to be designed to recover the allowable waste heat output without dropping the flue-gas temperatures below the condensation point.

In this temperature region the conversion efficiency is quite low. Maximum efficiencies commonly available for low-temperature thermoelectric materials are approximately $5 \%$.

\section{Construction of a prototype}

A prototype of the thermoelectric generator was designed and produced at the Energy Institute, Brno University of Technology. This generator was intended to verify the possibility of recovering waste heat from flue gas. A Verner A251.1 automatic biomass boiler served as the heat source. The scheme of our micro-scale CHP unit is shown in Figure 5. The design of the thermoelectric generator is shown in Figure 6. The most important parameters of this unit are shown in Table 1.

Table 1: Design specification of the prototype biomass thermoelectric boiler

\begin{tabular}{|l|l|}
\hline Parameter & \\
\hline Rated Heat Input & $25 \mathrm{~kW}$ \\
\hline Fuel Heat Input Range & $7.5-30 \mathrm{~kW}$ \\
\hline Minimum Return Water Temperature & $60^{\circ} \mathrm{C}$ \\
\hline Hot Surface Temperature & $220^{\circ} \mathrm{C}$ \\
\hline Nominal Electrical Power Output & $100 \mathrm{~W}_{\mathrm{el}}$ \\
\hline Fuel & Wood Pellets \\
\hline
\end{tabular}

This prototype was constructed with industrially available low-temperature thermoelectric material which allows operating temperatures of up to $230^{\circ} \mathrm{C}$. Each thermoelectric module consists of 127 thermocouples made from bismuth telluride.

\section{Application market}

The inadequate efficiency of state-of-the-art thermoelectric materials prevents the utilization of mass waste heat. In future, new materials and further increases in energy efficiency are expected, and the time will come when thermoelectric technologies reach a level that can support a power generation market [6].

\section{Conclusion}

Thermoelectric generators can convert thermal energy directly into electricity. These solid state devices are based on the Seebeck effect. A typical thermoelectric generator is composed of a large number of semiconductor thermocouples. The potential of a material to extract heat and convert it into electricity is quantified by the non-dimensional figure-of-merit $Z T$. 


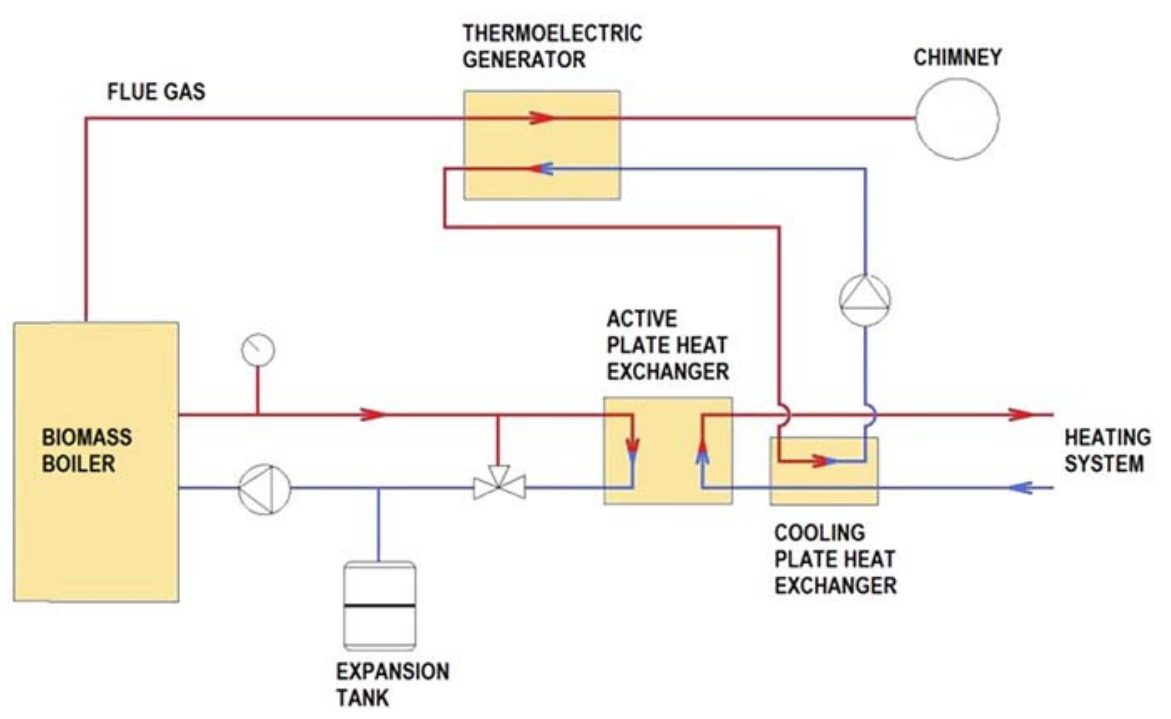

Figure 5: Scheme of the prototype biomass thermoelectric boiler

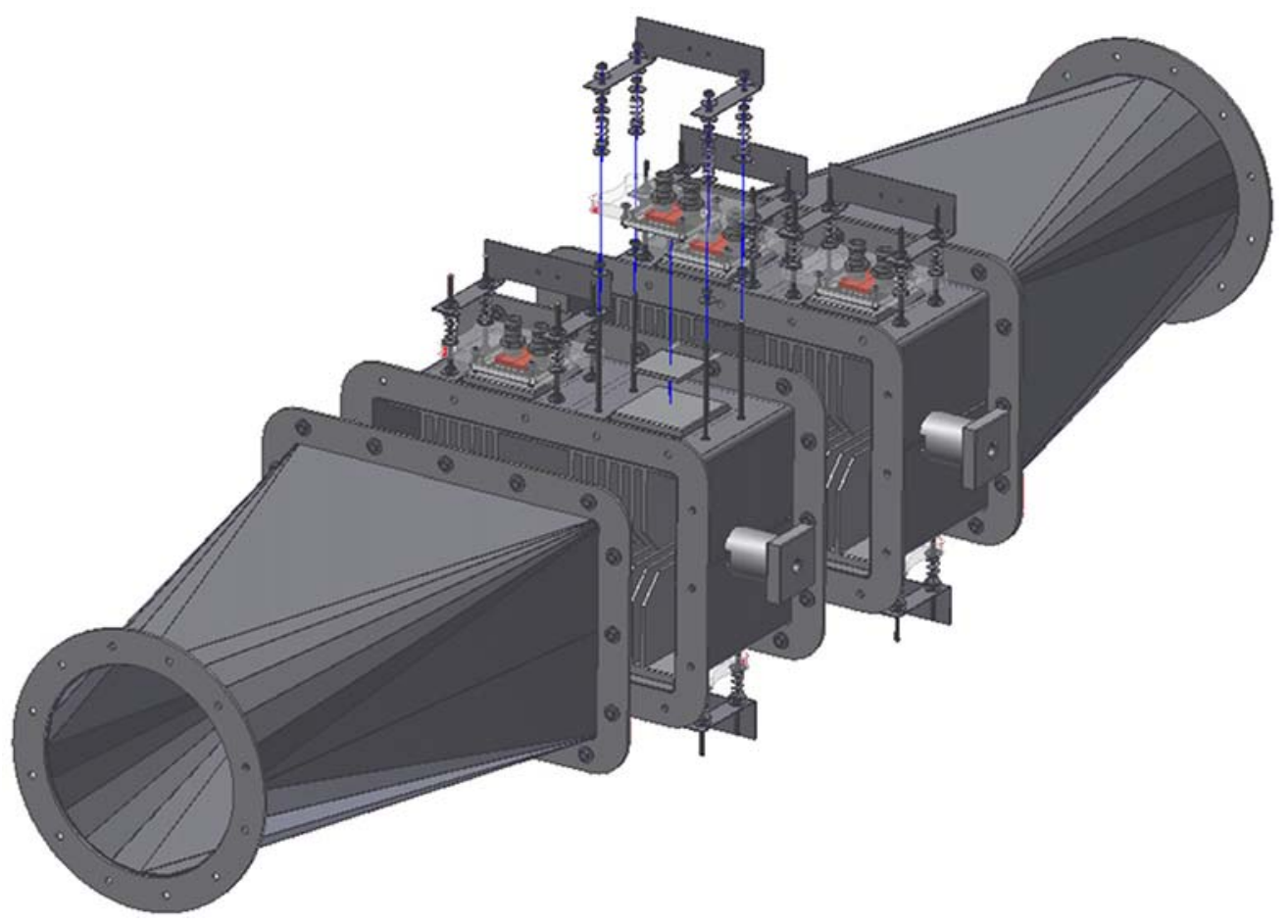

Figure 6: Design of the prototype thermoelectric generator

Modern automated combustion systems need to be connected to the electricity grid. Boilers with an integrated thermoelectric generator can utilize waste heat from the furnace. The CHP units that are created provide an independent source of electric energy and enable efficient use of fuel.

\section{Acknowledgement}

This work has been financially supported by the Brno University of Technology under Grant BD13101004.

\section{References}

[1] Rowe, D. M.: CRC handbook of thermoelectrics. 1st ed. CRC Press, 1995. 701 s. ISBN 978-0849301469.

[2] Jing-Feng, L., Wei-Shu, L., Li-Dong, Z., Min, Z.: High-performance nanostructured thermoelectric materials, NPG Asia Mater. 2(4), 152-158, 2010. Published online 21 October 2010.

[3] Rowe, D. M. (ed.): Thermoelectrics handbook: macro to nano. 1st ed. [Boca Raton] : CRC Press, 2006, 1014 s. ISBN 978-0849322648. 
[4] Riffat, S. B., Ma, X.: Thermoelectrics: a review of present and potential applications, $A p$ plied Thermal Engineering, 23, 913-935, 2003.

[5] Höftberger, E., Moser, W., Aigenbauer, W., Friedl, G., Haslinger, W.: Grid autarchy of automated pellets combustion systems by the means of thermoelectric generators, Bioenergy2020+ [online]. 2010, 26 s. [cit. 2012-03-16]. http://www.bioenergy2020.eu/files/publications/ pdf/I1-2481.pdf

[6] Kawamoto, H.: R\&D trends in high efficiency thermoelectric conversion materials for waste heat recovery. Quarterly review [online]. 2009, 30, [cit. 2012-03-16]. s. 54-69. http://www.nistep.go.jp/achiev/ftx/eng/stfc/ stt030e/qr30pdf/STTqr3004.pdf 